\section{$\underset{\substack{\text { hommes } \\ \text { \& migrations }}}{ }$}

\section{Hommes \& migrations}

Revue française de référence sur les dynamiques

migratoires

1282 | 2009

Santé et droits des étrangers : réalités et enjeux

\title{
Entretien avec Karim Kal
}

\section{Isabelle Renard et Karim Kal}

\section{CpenEdition \\ Journals}

\section{Édition électronique}

URL : http://journals.openedition.org/hommesmigrations/468

DOI : 10.4000/hommesmigrations.468

ISSN : 2262-3353

\section{Éditeur}

Musée national de l'histoire de l'immigration

\section{Édition imprimée}

Date de publication : 1 novembre 2009

Pagination : 164-171

ISSN : 1142-852X

Référence électronique

Isabelle Renard et Karim Kal, «Entretien avec Karim Kal », Hommes \& migrations [En ligne], 1282 |

2009, mis en ligne le 29 mai 2013, consulté le 24 septembre 2020. URL : http://

journals.openedition.org/hommesmigrations/468; DOI : https://doi.org/10.4000/hommesmigrations. 468

Ce document a été généré automatiquement le 24 septembre 2020.

Tous droits réservés 


\title{
Entretien avec Karim Kal
}

\author{
Isabelle Renard et Karim Kal
}

ISABELLE RENARD : pouvez-vous nous parler de votre parcours et plus particulièrement du choix du médium photographique?

Karim Kal : j'ai suivi une première formation à l'école d'art d'Avignon de 1996 à 1998 avant d'intégrer l'École supérieure d'art de Grenoble, où enseignait Jean-Luc Moulène. Le choix n'était pas dicté uniquement en terme de medium, mais était lié à des engagements de gauche auxquels j'étais très sensible. Malheureusement, Jean-Luc Moulène est parti après quelques semaines, en résidence au Japon, et je n'ai pas eu l'occasion de travailler beaucoup avec lui.

Enfin, à l'issue des Beaux-Arts, j'ai effectué un diplôme à l'école de photographie de Vevey en Suisse. Cela m'a donné l'occasion de travailler avec des praticiens de la photographie et d'avoir un aperçu des possibilités techniques à explorer. C'est une école très réputée dans le cadre de l'histoire de la photo.

La photo est un medium qui m'intéresse par son histoire tout à fait singulière, qui s'est développée parallèlement à l'histoire de l'art. Ensuite, à partir de Man Ray et des années trente, la photo a commencé à investir le champ de l'art. Ainsi, une histoire de l'art photographique nous laisse la possibilité de "discuter" avec d'autres champs historiques et artistiques. Il y a de vrais enjeux, spécifiques à ce medium pratiqué par de nombreuses personnes tant à un niveau professionnel qu'à un niveau amateur. La frontière est très ouverte. La photographie est un medium avec lequel je ressens beaucoup d'affinités. Personnellement, j'ai tout type de matériel, numérique et argentique, dont je me sers suivant les besoins et les projets.

Quel est le sens de votre démarche? De manière générale, vous interrogez les notions de mouvements migratoires et d'identité territoriale?

La question de l'identité territoriale m'intéresse énormément, elle est tout à fait centrale dans ma recherche. Pour ce qui est de la question des mouvements migratoires, elle est induite par ce premier questionnement sur l'identité territoriale. Je crois que le fond de cette énergie vient du fait d'avoir grandi dans une région - la Haute-Savoie - qui est assez provinciale et rurale dans un pays très centralisé comme la France. Avoir grandi à la campagne, tout en baignant dans une culture ou contre- 
culture urbaine, m'a sans conteste influencé. Cela provoque un sentiment de marginalité. Au-delà du fait d'avoir des parents d'origines différentes, je crois que ce sont davantage les questions de la ruralité et de l'urbanité qui m'ont guidé vers ces notions d'identité liées à un territoire.

Votre père est Algérien, votre mère Française. Votre travail est le reflet de ce que vous nommez vous-même un "besoin d'ancrage historique de votre propre destinée". Votre propre histoire, votre parcours familial influencent-ils votre dynamique créatrice?

Oui, je pense que la plupart des personnes issues de l'immigration qui ne sont pas directement immigrées ont un travail de rééquilibrage, en terme d'identité, à effectuer. Paradoxalement, nous affirmons notre singularité tout en nous inscrivant dans la logique générale de la société. Cette idée d'affirmation est, je crois, particulièrement importante pour les jeunes de ma génération.

Cela a une incidence significative ; c'est un parcours de vie particulier et propre aux populations immigrées venues en masse en tant que main-d'œuvre non qualifiée jusque dans les années soixante-dix. Il y a des fantasmes sur les populations immigrées d'origine musulmane, sur le fait qu'elles sont en mesure ou non de s'adapter aux codes sociaux, de s'insérer dans le tissu économique et culturel, etc. Tout en allant à la même école républicaine, en ayant les mêmes outils et les mêmes atouts, les enfants issus de l'immigration se trouvent à la sortie du système scolaire avec des problèmes qui sont ceux de leurs parents, de leur génération, sans qu'ils soient forcément les leurs. Nous ressentons le même regard porté sur nos parents qui se reflète sur nous. Pour ma part, pendant mon enfance, j'ai souffert du racisme qui existait autour de moi, mais pas pour des actes qui m'étaient directement destinés. Même si, pendant mon enfance, je n'étais pas entouré spécialement d'intellectuels ouverts, je n'ai jamais développé de sentiment d'exclusion ou de désir de repli communautaire. La perception de ce type de regard est venue plus tardivement.

Vous parlez de parcours de vie qui se croisent ; le vôtre, celui de vos parents...

Culturellement, c'est très compliqué. Ma mère est Française. Mon père, Algérien, ne m'a jamais tenu un discours d'exclusion, il a toujours essayé de me préserver de tout problème d'exclusion dont j'aurais pu pâtir en raison de mes origines. Mes parents ne m'ont jamais poussé à faire des choix religieux ou culturels. Je n'ai jamais eu l'impression de porter un quelconque message d'une communauté au reste de la société. On m'a incité ensuite à le faire, à l'école, où j'ai remarqué une curiosité de la part du corps professoral par rapport au vécu d'un enfant d'immigré. Beaucoup de gens essayaient de valoriser cela, mais j'ai voulu me protéger et éviter de me positionner en fonction de mes origines.

Dans un entretien réalisé par Michel Poivert en février 2008, vous vous remémorez votre enfance et vous parlez à propos de l'Algérie de "l'autre part de votre culture, de vos origines". II ne s'agit pas tout à fait du sentiment de "double absence", mais vous sentezvous partagé ? Ressentez-vous une part manquante?

Oui, très fort, pour la simple raison que j'ai de la famille proche en Algérie, qui n'a pas toujours la possibilité de venir en France, mais avec laquelle j'ai des contacts réguliers. C'est très compliqué à cause des visas, et très douloureux à vivre, car j'ai des cousins du même âge que moi et c'est un grand déficit pour eux de ne pas pouvoir venir en voyage en France. J'y vais souvent, depuis l'âge de 18 ans, mais cela fait un petit moment que je n'y suis pas retourné. À l'époque des troubles et des attentats, il 
est devenu particulièrement difficile de se rendre là-bas et, pour une série de raisons, je n'ai pas pu y aller pendant des années.

La CNHI a acheté en 2005 I'une de vos œuvres, Images d'Alger 2002. Pouvez-vous nous la décrire et nous dire quel a été le point de départ de ce travail ?

À travers mon travail, je questionne les mouvements migratoires, les notions de frontière, d'étrangeté... Mon outil principal est la photographie, et mon matériau la ville, le bâti.

À partir de 1997, alors étudiant aux Beaux-Arts, j'ai fait de fréquents voyages à Alger. Il s'agissait de voyages de plaisir certes, mais aussi d'une véritable période d'apprentissage culturel, de familiarisation aux codes sociaux et d'imprégnation de ce qui était mon pays au même titre que la France. C'était une sorte de réapprentissage de ce que j'avais toujours eu en moi sous forme instinctive. À l'époque, c'était un travail très long et très précieux pour moi, je ne voulais pas le raconter, mais, en même temps, il y avait quelque chose d'urgent qui se mettait en place en moi. On était en pleine époque du terrorisme, le climat pour le travail d'un photographe français était très compliqué et la réalité humaine terrible. Je me suis alors posé la question de savoir quelles images je pouvais réaliser d'Alger et des Algériens dans l'optique de les montrer en France. La grande majorité des visuels circulant dans les médias hexagonaux témoignait des atrocités terroristes ou, à de rares occasions, décrivait la situation sociale très difficile de cette période. Sans nier la nécessité de tels discours, ni leur véracité, ils me semblaient ne pas suffire. Ils faisaient abstraction de l'essentiel de ma propre expérience de l'Algérie.

C'est pourquoi j'ai voulu des images qui respirent, équilibrées, presque apaisées. Des images ouvertes sur l'ailleurs, transcrivant les éléments qui m'ont le plus frappé en Algérie: l'ouverture d'esprit, la conscience de l'autre, l'intérêt partagé pour la géopolitique... Quel décalage entre la connaissance que j'avais des réalités algériennes avant mes voyages et la conscience que les gens de mon âge avaient de ma vie quotidienne en France! Pour regarder le monde et le comprendre, Alger offrait une situation bien plus favorable que la mienne.

La question du départ est sous-tendue bien évidemment dans ma pièce comme faisant partie des choix de vie de chacun. D'autant plus avec la guerre. Et la situation géographique de la ville n'y est pas étrangère non plus, telle qu'elle est en littoral, tournée vers l'Europe. Dans les cadrages et les compositions, mes images voulaient malgré tout laisser des fragments, des indices permettant une lecture sociale ou événementielle de la situation. La partie basse de l'affiche, en effet, montre deux ruelles qui ont été le théâtre des inondations qui avaient frappé le quartier de Bab El Oued et ses habitants peu de temps auparavant.

Images d'Alger 2002 est un peu une œuvre oxymore. Elle est fondée sur une antinomie. Elle s'ouvre sur la Méditerranée, sur cette immensité bleue, marine et céleste. C'est une œuvre qui apaise, une œuvre qui invite au rêve. Et paradoxalement, elle a été réalisée à la fin de la guerre civile algérienne. Un décryptage plus approfondi des images nous ramène à une réalité plus difficile.

Ces images ont été pensées pour être diffusées en France, en contrepoint des images de presse de l'époque. Le récepteur de ces photographies était très clairement identifié. Je voyais et rencontrais en Algérie des gens de grande force, qui manifestaient leur volonté de continuer leur travail et de s'occuper de leur famille malgré tout. Ils manifestaient leur volonté de continuer à vivre, sourire, accueillir les 
autres. Il fallait laisser la place à cela, montrer cette sérénité face aux difficultés que les Algériens ont rencontrées à cette période.

Tenter de montrer ce que les médias ne dévoilent pas forcément, aller au-delà... est-ce une sorte de réappropriation de l'information à travers votre propre prisme? Votre approche se frotte au documentaire pour le dépasser et offrir une œuvre souvent allégorique. Votre démarche se rapproche-t-elle du journalisme, avec une dimension plastique?

Je ne pense pas que ma démarche dépasse l'acte journalistique. J'ai simplement ressenti une injustice, un déséquilibre entre la prédominance d'images sur la difficile situation de l'Algérie par rapport à cette force des Algériens que je ressentais lors de mes séjours là-bas. Il y avait un véritable déséquilibre et cette expérience a été pour moi initiatique.

\section{Comment votre travail a-t-il été reçu en Algérie?}

Il a été montré à l'occasion de l'année de l'Algérie, en collaboration avec des instances culturelles algériennes, et il a été apprécié de manière diverse par les acteurs culturels et artistiques présents.

Cette posture poétique a peut-être été interprétée à l'époque pour de la naïveté ou de l'angélisme, notamment par ceux qui pensaient que l'on ne pouvait pas faire l'économie d'un propos sur le terrorisme dans le pays. Malgré tout, je crois que l'Algérie souffrait d'un véritable déficit d'image. Je n'ai pas eu la prétention de changer les choses, mais j'ai proposé un autre regard qui pouvait en même temps être un point de vue recevable sur les réalités algériennes.

Ce qui est intéressant, c'est que vous essayez de dépeindre l'espace du spectateur, lequel n'est pas sur l'image mais suggéré. Les cadrages et les compositions évoquent ce qui ne se voit pas: le regard des Algérois, porté vers la mer, vers l'ailleurs. Les images frontales, qui deviennent presque des aplats de couleur, nous emportent dans la sphère symbolique.

Ce qui m'a frappé lors de mes séjours, c'est la pertinence du regard des Algérois sur mes réalités quotidiennes françaises. Cette pertinence contrastait fortement avec la vision limitée que l'on pouvait avoir des leurs, depuis l'Europe.

La communication univoque sur l'Algérie fait du mal au pays. L'image est fragmentée, hyperfocalisée, mais elle nous semble générale.

Que pensez-vous de la place de cette pièce au sein de la collection permanente du musée de la Cité ?

Je pense que l'intérêt de cette pièce tient essentiellement à son point de vue subjectif, inclus dans la question du départ. Elle invite à une recontextualisation de l'envie ou de la nécessité migratoire. Elle constitue probablement un contrepoint intéressant à d'autres travaux présents dans la collection.

Vous servez-vous de certains codes de l'image de presse?

Je ne suis pas en opposition avec les codes de la presse. Dans le cas précis des images d'Alger, c'était exactement cela, mais ce n'est pas ma logique de travail ou ma démarche en général. Cela m'intéresse, mais ce n'est pas mon cheval de bataille. La situation a fait que j'ai été confronté à une représentation ponctuelle de ce pays, que je voulais rééquilibrer.

Au-delà de ce que vous donnez à voir, c'est toute la question de la représentation et des modes de représentation photographiques qui sont en jeu.

Cela s'est fait de manière intuitive. J'avais la volonté de montrer la force que j'avais perçue et l'urgence de se soustraire à une vision conventionnelle. Effectivement, 
j'avais ces idées-forces en tête et j'ai donc senti intuitivement comment ces images devaient être. Je ne crois pas avoir réfléchi en priorité au cadrage. Cela est venu après, comme, par exemple, le choix de la composition avec les quatre photographies. La forme s'est imposée d'elle-même. La photographie est un outil de communication et de conditionnement, et il est important de bien réfléchir à l'emploi de ce medium. Sa vocation même est de véhiculer de l'information. La photo a un rapport au réel qui est radical, ce qui en fait un moyen essentiel pour se positionner sur les questions de société.

Dans mon approche de la photographie, il faut que l'image que je ramène porte les informations nécessaires. Les évocations ne m'intéressent pas. S'il y a évocation dans cette pièce, c'est à cause de la mer: la puissance de cette présence appelle effectivement au voyage.

Il faut resituer l'œuvre dans son contexte et dans son époque. C'est une œuvre contemporaine et je suis heureux qu'elle soit présentée à la Cité. Ce qui m'intéresse, c'est que l'image ne soit pas présentée dans une surenchère de dramatisation. Elle s'inscrit au contraire dans des trajectoires de vies.

Je suis fier qu'Images d'Alger 2002 soit dans cette structure et participer aux activités éducatives me semble important.

Je pense qu'il faut une double action, à la fois scientifique, dans un vif dialogue entre l'institution et le public, et individuelle, à partir de témoignages personnels.

Précisément, que pensez vous du choix de l'art contemporain dans ce musée d'histoire?

C'est très bien que l'art contemporain soit présent dans un lieu qui n'est pas, à la base, conçu pour cela.

Les réflexions sur l'immigration et son incidence sur la société française sont très présentes dans les productions des artistes français - j'entends par "français" qu'ils vivent ou travaillent en France. Les angles diffèrent du documentaire. Ils proposent souvent des pistes de lecture qui humanisent et sensibilisent des idées que la photographie de reportage, entre autres, - de par sa large diffusion, ses codes partagés, sa longue et riche histoire - a probablement un peu banalisées.

Je pense, par exemple, aux tampons de visa surdimensionnés de Barthélémy Togo, à leur implacable puissance, à leur mélange de violence et d'incongruité. Cette œuvre me semble d'une autre générosité, d'une autre efficacité que les images que nous consommons quotidiennement à travers la presse. Ce genre de pièces nous conduit à nous reconsidérer en tant que public. Ces œuvres nous déséquilibrent, nous déstabilisent et, finalement, elles nous font vivre une réalité que nous avons l'habitude de regarder à distance.

Vous considérez-vous comme un artiste engagé ?

Oui, c'est important pour moi, d'autant plus que les questions liées au social traversent ma recherche. Être plasticien implique le fait d'avoir une certaine sensibilité pour se saisir de la réalité et tenter de la retranscrire dans un espace de liberté. 
Vos principaux sujets sont le bâti, l'urbanisme, envisagés comme des marqueurs culturels et politiques. Pouvez-vous nous parler des travaux réalisés à Alger sur les cités d'urgence et à Cayenne en Guyane?

En ce qui concerne ma série Cités d'urgence réalisée en 2006, l'impulsion a été donnée de la même manière que pour Images d'Alger. Il s'agit d'une réflexion générale sur les représentations photographiques diffusées dans la presse et sur l'identification d'une tendance esthétique face à des évènements internationaux. L'enjeu est de me situer face à cette tendance, en en imitant les codes et en les transposant sur un sujet qui, pour moi, nécessite d'être mis en relation avec ces évènements.

En 2003, les États-Unis attaquent l'Irak et, pendant trois semaines, un mois, tous les magazines internationaux diffusent des images de nuit des bombardements sur Bagdad. Fin 2005, des émeutes ont lieu en banlieue parisienne et l'on diffuse des images de nuit proches de celles de Bagdad. La presse nationale et internationale fait ses gros titres sur ces deux évènements n'ayant pas grand-chose en commun. Ils bénéficient cependant, de manière surprenante, d'un traitement quasi équivalent et de systèmes de représentation très proches. Au début de l'année 2006, je suis parti en Algérie faire des photos des cités HLM commandées par la mairie Chevallier pendant la guerre d'Algérie afin de vider les bidonvilles et de reloger certains musulmans. Cela a été fait en catastrophe afin de contenir les mouvements populaires. 4500 logements de $15 \mathrm{~m}^{2}$ sans sanitaires pour la seule cité Climat de France ont donc été construits pour loger des familles entières.

Il s'agit de grands ensembles sociaux construits en période de guerre. Du bâti comme opération militaire. Le lien historique entre le logement social et la guerre est donc situé au sein même de ces cités d'urgence à Alger.

De plus, les architectes français (Pouillon, Simounet, Daure, Béri...) qui ont construit ces ensembles HLM en Algérie en pleine guerre d'indépendance sont rentrés en France et ont édifié, dans les années soixante, nombre de grands ensembles du même type autour des grandes villes françaises. Des grands ensembles qui ont été, pour certains, le théâtre des émeutes de 2005.

En ce qui concerne le projet de Cayenne, j'ai reçu en 2004 une bourse de la DRAC pour aller faire des photos en Guyane. Le projet portait sur la résurgence de la forme coloniale dans l'architecture contemporaine. La question coloniale m'interpelle. Cela m'a poussé naturellement vers les territoires d'outre-mer. Or, à mon arrivée, en faisant le tour des quartiers populaires, des ensembles HLM, des bidonvilles de la périphérie, j'ai été frappé par l'extrême pauvreté d'un grand nombre d'habitants de la ville. J'ai donc très vite réorienté mon projet vers ces territoires.

Parmi ces lieux, un grand ensemble s'appelle "La Rénovation". Il y a une forme de cynisme par rapport aux projets architecturaux. Ce sont des transpositions directes de l'habitat social métropolitain, sans prise en compte des réalités locales. Cela renseigne sur les intentions et sur le niveau de réflexion par rapport aux besoins locaux.

Mon travail est une réflexion plastique sur l'aspect visuel des constructions. Des structures comme les constructions de type favelas sud-américaines, malgré leur aspect dépouillé, sont l'expression des désirs des habitants, et, bien entendu, de leurs moyens. Les gens existent plus à travers ces structures qu'à travers les ensembles HLM. 
J'ai donc tenté de travailler quasiment avec une approche de portraitiste. C'est pour cette raison que les images sont souvent prises de trois-quart, avec un angle où la structure est clairement isolée de son contexte ou s'en détache. Les constructions sont toujours entourées d'un élément de l'environnement. Cela permet de délimiter ainsi des éléments qui balisent cet environnement, comme une voiture ou un tas de détritus, et qui participent à la structure même de l'image. Dès lors, les grands ensembles, pour lesquels l'enjeu a été d'en traduire la radicalité, existent presque comme décors de théâtre par leur statut, leur taille, alors que les petites constructions sont individualisées et entretiennent un dialogue avec leurs habitants.

Quels sont vos prochains travaux?

Deux lignes claires se dégagent aujourd'hui de mes recherches, s'intéressant chacune à la question du territoire. L'une m'emmène vers les représentations des situations singulières historiques, là où les territoires se sont faits, défaits, rattachés ou autonomisés... L'autre me porte vers la question de l'individualisation de certains territoires, leur réappropriation, leur fonctionnalisation.

J'ai actuellement un projet en Martinique, où j'espère pouvoir me rendre courant 2010, et un autre projet dans les Alpes, en Savoie, en Haute-Savoie et en Isère, sur le logement social de moyenne montagne, qui se fera vraisemblablement en 2010.

\section{RÉSUMÉS}

Karim Kal est né en 1977 à Genève, de père algérien et de mère française. Il vit et travaille en France. Une démarche identitaire et un enracinement culturel multiple commandent sa recherche. Son travail se veut ainsi le reflet de ce qu'il nomme lui-même un "besoin d'ancrage historique de sa propre destinée" et se traduit par une œuvre souvent allégorique.

\section{AUTEURS}

\section{ISABELLE RENARD}

Chargée de mission Art contemporain, Musée national de l'histoire et des cultures de l'immigration 